\title{
Realistic Near-Term Propellant Depots: Implementation of a Critical Spacefaring Capability
}

\author{
Jonathan A. Goff ${ }^{1}$ \\ Masten Space Systems, Inc., Mojave, CA, 93501 \\ Bernard F. Kutter ${ }^{2}$ and Frank Zegler ${ }^{3}$ \\ United Launch Alliance, Littleton, CO, 80127 \\ Dallas Bienhoff ${ }^{4}$ \\ The Boeing Company, Arlington, VA, 22202 \\ Frank Chandler ${ }^{5}$ \\ The Boeing Company, Huntington Beach, CA 92647 \\ Jeffrey Marchetta ${ }^{6}$ \\ The University of Memphis, Memphis, TN, 38152
}

\begin{abstract}
Orbital cryogenic propellant depots and the ability to refuel spacecraft in orbit are critical capabilities for the expansion of human life throughout the Solar System. While depots have long been recognized as an important component of large-scale manned spaceflight efforts, questions about their technology readiness have so far prevented their implementation. Technological advancements in settled cryogenic handling, passive thermal control systems, and autonomous rendezvous and docking techniques make near-term implementation of cryogenic propellant depots significantly more realistic. Current work on flight-demonstration tools like ULA's CRYOTE testbed, and Masten Space Systems's XA-1.0 suborbital RLV provide methods for affordably retiring the remaining technical risks for cryogenic depots.
\end{abstract}

Recent depot design concepts, built on high-TRL technologies and existing flight vehicle hardware, can enable easier implementation of first-generation propellant depots without requiring extensive development programs. Some concepts proposed by industry include disposable "pre-depots", single-fluid simple depots, self-deployable dual-fluid single-launch depots using existing launchers and near-term launcher upgrades, and multi-launch modular depots. These concepts, particularly the dual-fluid single-launch depot enable robust exploration and commercial transportation throughout the inner Solar System, without the need for HLVs, while providing badly-needed markets to encourage the commercial development of more affordable access to space.

\footnotetext{
${ }^{1}$ Propulsion Engineer, 1570 Sabovich St. Bldg 25, AIAA Member.

${ }^{2}$ Sr. Staff, Manager Advanced Programs, P. O. Box 277005 MS U9115, AIAA Senior Member

${ }^{3}$ Sr. Staff, Advanced Programs, United P.O. Box 277005 MS U9115, AIAA Member

${ }^{4}$ Manager, In-Space \& Surface Systems, Advanced Space Exploration, 1215 S. Clark St. MC 793C-G042, AIAA Senior Member.

${ }_{6}^{5}$ Director, Propulsion \& Cryogenic Technologies, 5301 Bolsa Ave/H012-2B201, Associate Fellow.

${ }^{6}$ Associate Professor, Mechanical Engineering, 322D Engineering Sciences Building Memphis, TN 38152, AIAA Member.
} 


\section{Nomenclature}

$\begin{array}{ll}M S S & =\text { Masten Space Systems, Inc. } \\ U L A & =\text { United Launch Alliance } \\ D A R P A & =\text { Defense Advanced Research Projects Agency } \\ L O_{2} & =\text { Liquid Oxygen } \\ L H_{2} & =\text { Liquid Hydrogen } \\ I S R U & =\text { In-Situ Resource Utilization } \\ I S S & =\text { International Space Station } \\ L E O & =\text { Low Earth Orbit } \\ E M L-1 & =\text { Earth-Moon L-1 LaGrange Point } \\ E M L-2 & =\text { Earth-Moon L-1 LaGrange Point } \\ H L V & =\text { Heavy Lift Vehicle } \\ R L V & =\text { Reusable Launch Vehicle } \\ T R L & =\text { Technology Readiness Level } \\ m T & =1 \text { Metric Ton }\end{array}$

\section{Introduction}

$\mathrm{O}$ $\mathrm{NE}$ of the key challenges of interplanetary space travel is the vast amount of propellant that has to be hauled from Earth to reach even the nearest bodies in the Solar System. This situation is not unique to spaceflightthere are many historical examples of groups dealing with the challenges of operating in hostile environments far from sources of food and fuel. Whether the challenge was crossing the frozen deserts of Antarctica with sled dogs and skis, exploring underwater caverns in Mexico or the US military trying to operate steam-powered naval vessels across the vast Pacific in the late 1800s, caches of supplies and fuel were a key part of solving transportation logistics problems. For Amundsen's South Polar expedition, caches of supplies and food were left at key points along the way to the South Pole ${ }^{1}$. For an underwater spelunking expedition in the Huautla Plateau of Mexico, the US Deep Caving Team established a series of supply camps at different dry points within the cavern ${ }^{2}$. For the US Pacific Fleet, the solution was naval coaling stations and colliers located in places like Pearl Harbor, Midway Island, Guam, and American Samoa.

The similarity of the interplanetary transportation logistics problem to its historical analogs was not lost on the pioneers of space travel in the $20^{\text {th }}$ century. As early as $1928^{3}$, scientists studying interplanetary travel began arguing that pre-positioning propellants in orbit would be required for any sustainable large-scale travel beyond Earth. Ever since those initial observations, the use of orbital propellant depots-facilities specifically designed to receive, store, and dispense propellants to visiting craft — has been an important part of many of the most ambitious transportation architecture proposals ${ }^{45}$, including the "tanker mode" concept for Earth Orbit Rendezvous favored by von Braun during the Apollo Program ${ }^{6}$. Perhaps one of the most valuable lessons learned from the Apollo Program was the difficulty of making even limited sorties beyond earth orbit without using propellant depots. Launching all of the supplies and propellant needed for exploration missions on just one rocket was only barely possible, and even then, it only permitted voyages of a couple of weeks, at the most. One could argue that the great expense of developing and operating the massive launch vehicles necessary for non-depot approaches is one of the principal factors which have prevented space exploration and development activities beyond LEO since the Apollo Program ended.

In recent years, a growing interest in commercial approaches to space transportation has led to the beginning of a renaissance in propellant depot efforts ${ }^{7}$. In addition to the logistical advantages, researchers have begun to notice economic benefits of orbital propellant depots. These benefits include providing demand for launch services that can help drive down launch costs by creating a robust and competitive marketplace, providing launch demand sufficient to encourage the development of commercial RLVs, enabling the reuse of in-space assets, and greatly increasing the utility of future ISRU efforts ${ }^{8}$. Depots have the potential to not only greatly reduce the difficulty of travel beyond LEO, but also to help promote the development of much more affordable and reliable means for accessing LEO in the first place.

However, in spite of over 80 years of recognition that orbital propellant depots are a critical necessity for affordable and sustainable travel beyond LEO, there has still been no serious effort to develop and field orbital propellant depots. In fact, many have opposed the idea. A large part of the opposition stems from two key misconceptions. The first of these misconceptions is that propellant depots are very technically risky and require 
large amounts of expensive R\&D work before we can even know if they will work, let alone before they can be implemented. The second key misconception is that orbital propellant depots imply an effort comparable in complexity to the International Space Station. Propellant depots, when viewed in this light, have often been seen as expensive and risky detours when compared to the HLV path taken by Apollo.

Several recent developments, however, indicate that propellant depots are much closer to reality than previously believed. While some key techniques still require demonstration, the fundamental technologies needed for initial propellant depot capability are already at a high level of maturity. Several innovative depot design concepts have recently been proposed, which have sufficient propellant storage capacity to enable near-term manned missions beyond LEO, while still being much simpler than previously envisioned approaches. In addition to concepts for simple near-term depots, several groups are now working on orbital and suborbital test-beds that will facilitate maturation of technologies needed for initial depot capabilities and continuous improvement of propellant depot technology. These new developments are realistic near-term options for developing and fielding these key pieces of space transportation infrastructure.

\section{Propellant Depot Technologies}

An important challenge in developing space systems and architectures is determining when and how to incorporate promising new technologies. On one hand, at least 80 percent of the life-cycle costs of a program are determined by decisions made during the conceptual design stage ${ }^{9}$. Being too conservative about incorporating new technologies can lock in high costs, threatening project sustainability. On the other hand, incorporation of new technologies carries technical risk, which often makes decision makers hesitant to adopt them. An example of this was the Apollo Program's eventual decision to use Lunar Orbit Rendezvous (LOR). Originally, orbital rendezvous was viewed as far too immature a technology to risk the program on. As one member of the committee that initially rejected the LOR approach put it, "We thought it too risky. Remember, in 1961 we hadn't even orbited Glenn yet. We certainly had done no rendezvous yet...it had to be dead right the first time. I mean, that just seemed like a bit much. ${ }^{, 10}$ In the end, however, LOR won out in spite of the fact that orbital rendezvous technology was then only at TRL 3 or 4 . The decision was largely motivated by the political need to avoid excessive life-cycle costs and development time for the massive Nova launch vehicle.

Propellant depot technologies now are significantly more mature than orbital rendezvous technologies were when they were selected as the baseline for Apollo's critical path. , Propellant depot technologies have benefited from decades of operational experience with cryogenic upper stages and technology maturation efforts such as the DARPA Orbital Express demonstrator. Many approaches, spanning a wide range of technological maturity, have been investigated for key depot functions, including cryogenic fluid management, propellant thermal control, and rendezvous, docking, and fluid coupling and transfer. . Some combinations of depot technologies can provide initial operational capabilities with very little additional technology demonstration, while some other interesting options will require more work. A program that utilizes incremental system demonstrations in parallel with a robust technology maturation effort can provide early, useful operational capabilities, while still allowing some of the promising but less developed technologies to be integrated as they reach a sufficient level of technological maturity.

\section{A. Microgravity Cryogenic Fluid Management}

The first key task of an orbital propellant depot is to store and handle fluids on orbit, particularly cryogenic propellants. Cryogenic fluids, such as $\mathrm{LO}_{2}$ and $\mathrm{LH}_{2}$, are stored and transferred daily on Earth for use in industrial and medical applications. Compared to the terrestrial environment, where cryogenic fluid handling is relatively simple, the microgravity environment in which orbital propellant depots operate presents many unique challenges. While the locations of liquids and gasses in a tank at rest in a gravity field are controlled and easily predictable, the behavior of the fluids in microgravity is more difficult to predict and presents some unique challenges for controlling the fluid flow. The processes of pressure venting and transfer of liquid propellant are complicated by the uncertainty of distribution of liquid and gasses within the tank. Lack of gravity-driven buoyancy effects makes thermal equalization within the liquid much more challenging than in even a slight gravity field. For non-volatile propellants, the options of using either elastomeric diaphragms or surface tension liquid acquisition devices to separate the liquid from the gas have been flight qualified. While there has been significant research on options for dealing with these challenges for cryogenic fluids ${ }^{11}$, the technology is less mature than for storable propellants. 


\section{Inertial Propellant Settling}

There are, however, alternatives to developing techniques for manipulating fluids in microgravity, which typically fall into the category known as settled propellant handling. Research for cryogenic upper stages dating back to the Saturn S-IVB and Centaur found that providing a slight acceleration (as little as $10^{-4}$ to $10^{-5} \mathrm{~g}$ of acceleration) to the tank can make the propellants assume a desired configuration, which allows many of the main cryogenic fluid handling tasks to be performed in a similar fashion to terrestrial operations ${ }^{12}$. The simplest and most mature settling technique is to apply thrust to the spacecraft, forcing the liquid to settle against one end of the tank. The thrust can come either from small rockets or from venting a small amount of boiled-off propellant gas through small nozzles and can be applied periodically or continuously.

Another proposed option, which ULA plans to flight-test in 2009 (on the DMSP-18 mission), involves

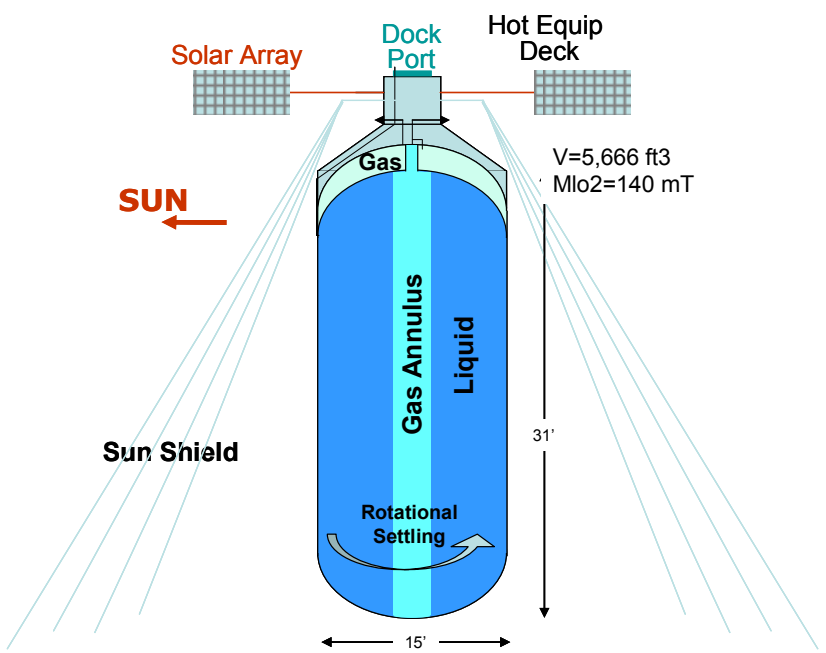

Figure 1. Using Axial Rotation to Provide Centrifugal Propellant Settling. Credit ULA spinning the tank about its axis, as shown in Fig. 1. In this technique, the propellant is forced against the side walls, leaving a core of gas along the axis of rotation ${ }^{13}$. This provides continuous settling without consuming boil-off gases.

\section{Tether or Gravity Gradient Settling}

Other options, which have not yet been demonstrated, use tethers or gravity gradients to provide the settling forces. One option, similar to the centrifugal settling option mentioned previously, is to extend a tether and a counterweight from a depot, and then to spin the assembly about its center of gravity. It is also possible to use the same configuration without spinning the assembly, by taking advantage of the gravity gradient. Any part of the assembly that is above the station's center of gravity is actually travelling faster than a separate object orbiting at that altitude, resulting in a slight acceleration outward, and any piece below the center of gravity experiences an acceleration downward towards Earth. In order to provide adequate settling forces, the assembly only needs to be a few hundred meters long. Another approach would use electrodynamic tethers ${ }^{14}$ both for propellantless depot reboost as well as for propellant settling.

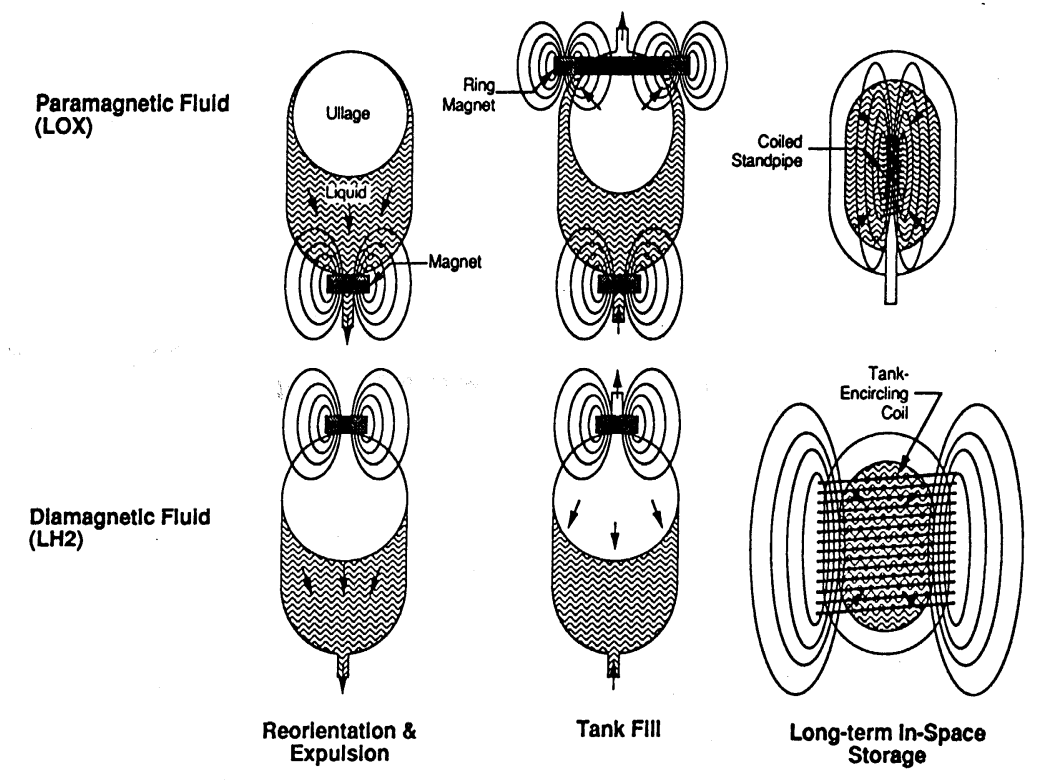

Figure 2. Magnetic Propellant Positioning Options for $\mathrm{LO}_{2}$ and $\mathrm{LH}_{2}$ Credit Jeffrey Marchetta 


\section{Electromagnetic Propellant Settling}

One particularly promising class of propellant settling options, shown above in Fig. 2, forces the fluid within the tanks to assume a desired orientation using strong permanent or electromagnets. The settling force is provided by the reaction of the inherent paramagnetic or diamagnetic properties of the propellants to an applied magnetic field ${ }^{15}$. $\mathrm{LO}_{2}$ is paramagnetic, meaning that it is slightly attracted to strong magnetic fields. $\mathrm{LH}_{2}$, methane, propane, and other light hydrocarbons are weakly diamagnetic, meaning they are repelled by strong magnetic fields. Because the bulk magnetic susceptibilities of the fuels are much lower than that of the $\mathrm{LO}_{2}-$ with $\mathrm{LH}_{2}$ being the least susceptible of the cryogenic fuels - the use of high temperature superconducting electromagnets may be required to make magnetic propellant positioning for these fuels feasible. If $\mathrm{LH}_{2}$ magnetic propellant positioning is feasible, then the same technique can be used for the more magnetically susceptible light hydrocarbons. At this moment, the technology for electromagnetic settling is still fairly early in its development. There have been some small experiments such as MAPO ${ }^{16}$, which have been flown on the NASA zero-gravity airplane (Vomit Comet). Coupled fluid/electromagnetic models were correlated to the experimental results ${ }^{17,18,19}$. Analytical work has begun this year using those models to determine the feasibility of using magnetic propellant positioning for tanks on the scale required for propellant depots. If magnetic propellant positioning can be perfected, it may provide many of the advantages of both settled and non-settled microgravity propellant handling as shown in Table 1.

Table 1. A Comparison of Cryogenic Fluid Handling Techniques

\begin{tabular}{|c|c|c|}
\hline Technique & Advantages & Disadvantages \\
\hline Zero-G Handling & $\begin{array}{l}\text { - Does not require reaction mass for } \\
\text { propellant settling. } \\
\text { - Integration with big stations easier } \\
\text { - Configuration and orientation } \\
\text { independent of operations } \\
\text { - Loading/offloading operations } \\
\text { identical }\end{array}$ & $\begin{array}{l}\text { - Zero-G thermal control, transfer, and } \\
\text { liquid acquisition are low TRL. }\end{array}$ \\
\hline Propulsive Settling & $\begin{array}{l}\text { - Settled cryo handling is high TRL, } \\
\text { and simplifies all other depot functions } \\
\text { - Settling and reboost functions can be } \\
\text { combined. }\end{array}$ & $\begin{array}{l}\text { - Uses reaction mass for settling } \\
\text { - Hard to integrate with existing space } \\
\text { stations } \\
\text { - Constrains tank arrangement to get } \\
\text { correct settling effects }\end{array}$ \\
\hline Centrifugal Settling & $\begin{array}{l}\text { - Does not require reaction mass for } \\
\text { propellant settling. } \\
\text { - Settled cryo handling is high TRL, } \\
\text { and simplifies all other depot functions }\end{array}$ & $\begin{array}{l}\text { - May require despinning for docking } \\
\text { - May need to be combined with another } \\
\text { process for transfer ops } \\
\text { - Constrains tank arrangement to get } \\
\text { correct settling effects }\end{array}$ \\
\hline ED Tether Settling & $\begin{array}{l}\text { - } \quad \text { Provides reboost and propellant } \\
\text { settling without using reaction mass } \\
\text { - Can use zero boil-off systems }\end{array}$ & $\begin{array}{l}\text { - Requires moderately large station with } \\
\text { significant solar power capability } \\
\text { - Low TRL for ED tethers } \\
\text { - Challenges docking } \\
\text { - Constrains tank arrangement to get } \\
\text { correct settling effects }\end{array}$ \\
\hline $\begin{array}{l}\text { Gravity Gradient } \\
\text { Settling }\end{array}$ & $\begin{array}{l}\text { - Does not require reaction mass for } \\
\text { propellant settling }\end{array}$ & $\begin{array}{l}\text { - Requires very long tether and large } \\
\text { overall system } \\
\text { - Complex system dynamics } \\
\text { - Constrains tank arrangement to get } \\
\text { settling effects correctly }\end{array}$ \\
\hline $\begin{array}{l}\text { Electromagnetic } \\
\text { Settling }\end{array}$ & $\begin{array}{l}\text { - Does not require reaction mass for } \\
\text { propellant settling. } \\
\text { - Provides more control over propellant } \\
\text { positioning. } \\
\text { - More flexibility on tank arrangements } \\
\text { and depot layout }\end{array}$ & $\begin{array}{l}\text { - Electromagnetic settling is low TRL } \\
\text { - Superconducting electromagnets may add } \\
\text { significant weight } \\
\text { - Uncertainty if existing electromagnets } \\
\text { sufficient for large } \mathrm{LH}_{2} \text { tank settling. }\end{array}$ \\
\hline
\end{tabular}


Propulsive propellant settling is a well understood process and can be easily incorporated into near-term propellant depots. As the more advanced inertial and electromagnetic approaches are technologically matured, they can be brought into service alongside of, or in place of, the first generation depots.

\section{B. Propellant Thermal Control}

The second task of an orbital propellant depot is to store the propellants for long durations with minimal or no boil-off losses. Due to the very low boiling points of cryogenic propellants, care must be taken to reduce the flow of heat into the propellants and remove the heat that does make it into the propellants. Current flight-demonstrated thermal control systems for cryogenic upper stages have not yet reached the efficiencies necessary for a reasonable propellant depot, however many of the insulation and cooling technologies have been developed to the point of preliminary ground-systems tests ${ }^{20}$.

\section{Thermal Insulation}

An important part of the thermal control involves isolating the propellants from internal and external heat sources. The main source of external heat load is, of course, the Sun itself. Planetary bodies, however, can also be significant sources of radiant heat for depots in low-orbits. There are also heat sources internal to the depot, such as electronics and power generation equipment, which must be isolated from the propellant section of the depot. For $\mathrm{LO}_{2} / \mathrm{LH}_{2}$ systems, the other main heat source is the temperature difference between the two propellants.

A wide variety of passive insulation techniques have been investigated ${ }^{21}$. Multi-Layer Insulation (MLI) material and deployable sunshades ${ }^{22,23}$ reduce the heat flux from external sources. Vacuum Insulation Panels (VIPs), which see extensive use in other industrial applications, and low-conductivity attachments, are also being considered for cutting down on heat flow between the tanks and between the tanks and the depot electronics. MLI has seen extensive operational use on satellites and space probes, and sunshades are becoming popular for space telescopes (e.g. Spitzer, Kepler and the James Webb Space Telescope), some of which need to maintain liquid helium temperatures at their optics for several years at a time ${ }^{24}$. Low-conductivity mounts are currently used in some places on upper stages, and vacuum insulation panels are being ground tested for eventual use on propellant tank common bulkheads and in other locations.

\section{Heat Rejection and Active Thermal Control}

Even the best insulation system, however, still allows some heat to flow into the propellants. The simplest method of dealing with the heat flow is to let the cryogen absorb the tank heating, resulting in a warming of the liquid. This is what is currently done on Centaur's $\mathrm{LO}_{2}$ tank, which is not vented on orbit. This method, however, is usable only for medium durations, because as the heat flow into the propellants, the pressure in the tank increases, which can eventually lead to tank rupture if unrelieved. Another approach for dealing with the heat flow into the propellants is to allow a small amount of the propellant to boil-off, and then vent the excess gas overboard, as has been and is done on Saturn S-IVB, the Centaur $\mathrm{LH}_{2}$ tank and the Delta IV upper stage. Not only does this process carry away excess heat from the propellant, and keep the tanks within their structural limits, but the process of venting the gases to space can also be used for propulsive settling of the propellants as well as for depot stationkeeping.

Some more advanced techniques have been proposed that would use the vented gas from the coldest propellant tanks to remove heat from the overall system. This is especially useful for $\mathrm{LO}_{2} / \mathrm{LH}_{2}$ systems, because the $\mathrm{LH}_{2}$ has over ten times the heat capacity of the $\mathrm{LO}_{2}$. It also starts off at a much cooler temperature, and makes up a much smaller fraction of the overall propellant mass. By using the $\mathrm{LH}_{2}$ boil-off to cool the $\mathrm{LO}_{2}$ tank, $\mathrm{LO}_{2}$ boil-off can be completely eliminated. This is much more mass efficient than allowing them both to boil and vent. It is also possible, using a Thermodynamic Venting System (TVS), to use the vented hydrogen gas to remove some more heat from its own tank. This is done by running the moderate-pressure boil-off gas through a Joule-Kelvin valve, which drops both the pressure and temperature of the vented gas, allowing it to provide some extra cooling for the $\mathrm{LH}_{2}$ tank before it is used for removing heat from the other tanks and subsystems in the depot. After cooling the $\mathrm{LO}_{2}$ tank, and the connection between the $\mathrm{LO}_{2}$ tank and the electronics section of the depot, the now much warmer gaseous hydrogen can be run through a nozzle to provide thrust for settling and station-keeping. While these passive boil-off venting techniques do result in the loss of valuable propellant mass, the amount of propellant lost to boil-off in a system with good thermal insulation may actually be less than the amount required for station-keeping. This means that unless some higher $I_{\text {sp }}$ or non-propellant means (such as an electrodynamic tether) is used for stationkeeping, the propellant loss to boil-off may actually be "free".

The other approach to heat rejection is using an active cryocooler to pump heat out of the propellants. Although almost all cryogenic fluid cooling in space to-date has been of the passive variety, there has been significant research 
into active cooling techniques due to their promise for zero boil-off operations. While zero boil-off depots are not required for initial operations, they represent a significant enabler of longer-duration deep space missions. They also make a lot of sense when used in conjunction with non-propulsive propellant settling where propulsive stationkeeping is not necessary. Active cryocooling eliminates the waste of propellants currently required for chill-down operations during transfer, increases system efficiency by eliminating boil-off of propellants over time, and makes it possible to keep the region around the depot clear of contamination. These benefits make cryocooling technologies well worth further development effort.

\section{Rendezvous, Docking, and Propellant Transfer}

The last major task of an orbital propellant depot is the transfer of propellant from visiting tankers into the depot and from the depot into customer spacecraft. This involves rendezvous and mating of the visiting vehicles, secure attachment of fluid couplings, and safe and efficient transfer of propellants through those couplings. This process was first demonstrated operationally by the Soviet space program in 1978 using storable propellants ${ }^{25}$ and is used routinely for propellant resupply of the ISS by the Russian Progress vehicle and the ESA Automated Transfer Vehicle. It has also been demonstrated recently in the US by the DARPA Orbital Express program, also with storable propellants.

While there has not yet been an operational demonstration of cryogenic propellant transfer between two vehicles on orbit, a similar process has been used to transfer propulsively-settled cryogenic propellants from the tanks to the engines for every multi-burn cryogenic upper stage since the Saturn S-IVB. The experience obtained from literally hundreds of such upper stage engine restarts - which are arguably even more finicky about two-phase flow than a depot-is a significant knowledge base from which propellant depot designers should be able to draw. The only piece of hardware that has not yet been flight demonstrated is the automatic connection of cryogenic fluid couplings on orbit.

The Orbital Express program, funded by DARPA and Boeing, demonstrated a number of key technologies which would be needed for a viable autonomous commercial propellant depot ${ }^{26}$. Of the technologies demonstrated, the ones most relevant to propellant depots were short- and long-range autonomous operations, including orbital rendezvous, proximity operations, spacecraft mating, autonomous connection of propellant couplings, and zero-g transfer of storable propellants. The Orbital Express system consisted of a large servicing vehicle, ASTRO (Autonomous Space Transfer and Robotic Orbiter), and a client satellite, NEXTSat (Next Generation Satellite), shown in Fig. $3^{27}$. The client satellite was mostly passive throughout the rendezvous and docking operations. All of the actuating mechanisms, such as the active coupling halves and the robotic arm, were located on ASTRO.

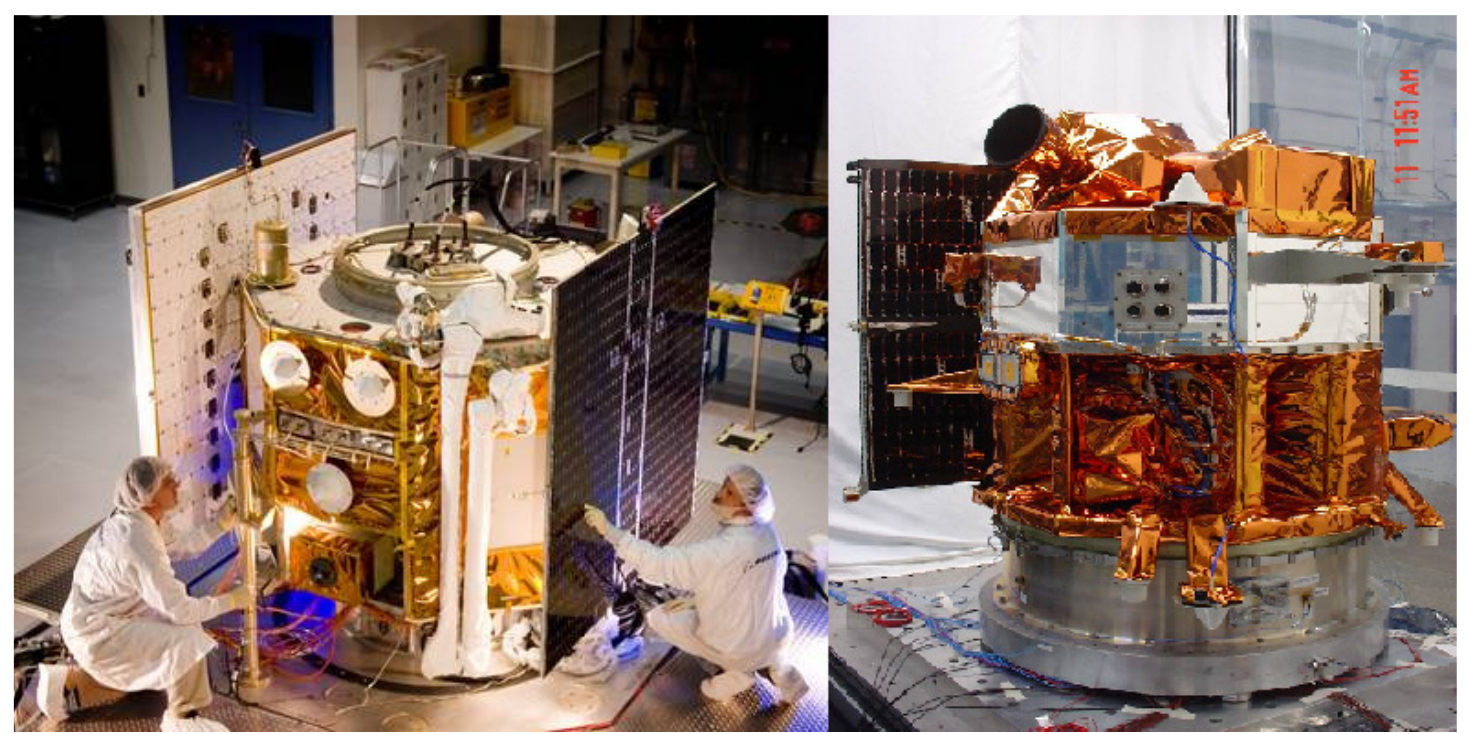

Figure 3. The Two Spacecraft of Orbital Express, ASTRO and NEXTSat. Credit DARPA, Boeing, and Ball Aerospace 
The propellant transfer system, Fig. 4, transferred storable propellants, including both hydrazine and pressurant gas $^{28}$, at ambient temperatures. The pressurant gas was transferred to provide ullage equalization and to demonstrate gaseous fluid transfer. There were 15 successful propellant transfers with varying ullage termination quantities up to and including a full tank load. These operations validated the essential techniques required for safe autonomous transfer of fluids on-orbit. While the couplings will probably be different for cryogenic fluid transfer,,the techniques and procedures demonstrated during the storable fluid transfer testing can used in cryogenic applications.

One promising approach to cryogenic fluid couplers, proposed by ULA for depot applications, is a coupling based on the slip-joint duct used in the $\mathrm{LO}_{2}$ feed line of the Atlas Centaur upper stage ${ }^{29}$. The slip joint duct, shown in Fig. 5, consists of two concentric tubes with a set of redundant cryogenic spring-energized Teflon dynamic seals and a set of low-friction guide bushings, all arranged in an annulus between the inner and outer tube. This system would be combined with shutoff valves on both sides of the connection to prevent leakage when the connection is broken. With proper design, this coupling can be self aligning, and the seal does not require large clamping forces to provide a leak-tight connection; only axial restraint is needed to resist pressure and fluid dynamic forces. The coupling also has a very low thermal mass, and can provide much larger flow area for a given sized coupling than other cryogenic couplings, resulting in faster fluid transfer times.
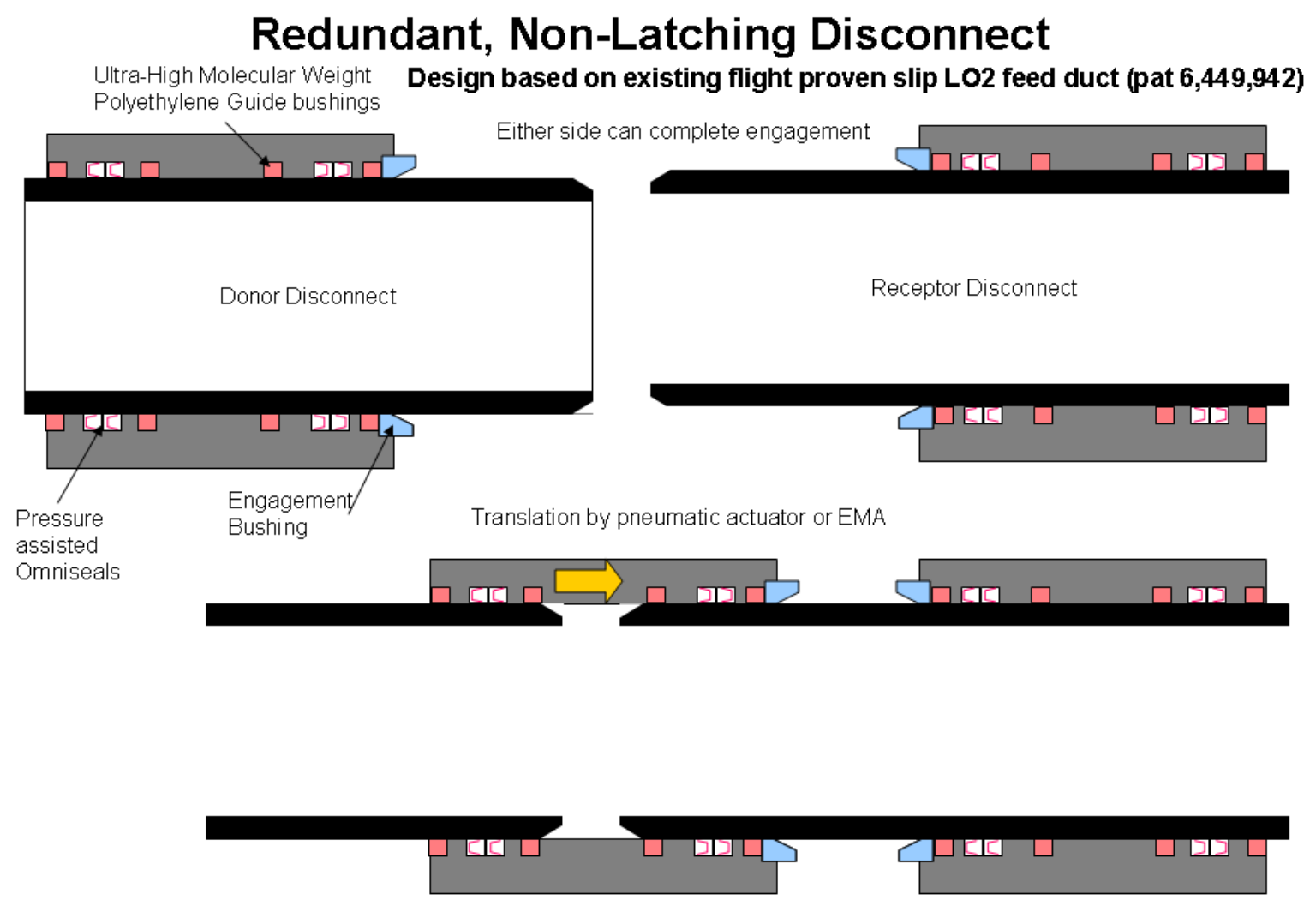

Figure 5. Illustration of a Slip-Joint Duct Connector for Cryogenic Propellant Transfer. Credit ULA 
Another contribution of the Orbital Express project to the development of propellant depots is the concept of using a space tug to simplify proximity ops and propellant transfer coupling connection. The main previous example of orbital propellant transfer, the Progress spacecraft, required the tanker vehicle to be a fully maneuverable proximity operations craft in its own right. This resulted in a very poor payload to spacecraft mass ratio — only $31 \%$ of the launched mass was usable payload. While it may be possible to design a more mass efficient tanker system based on the COTS cargo vehicles, and to minimize tanker "over head" performance loss through efficient tanker design $^{30}$ or by directly integrating the fluid load into the upper stage tanks, the parasitic mass of tanker docking and transfer systems still have to be taken into consideration for any depot architecture design.

However, the success of the Orbital Express program suggests that the delivered propellant mass fraction can be further increased by offloading most of the rendezvous and docking hardware to a reusable space tug ${ }^{31}$. Such an orbital tug system, when combined with an upper stage capable of station-keeping, would require only very minimal hardware on the delivery tanker. In operation, such a tug could leave the depot, rendezvous with the tanker and upper stage, mate to the tanker, remove the tanker from the upper stage, haul the tanker to the depot, attach propellant feedlines, and then dispose of the tanker after all the propellant has been transferred. This would allow the tanker to be a lot simpler, requiring only passive mating and propellant coupling hardware, and some simple controls. Offloading the expensive "smarts" to a reusable tug, which remains in orbit between missions, should reduce the cost of delivery tankers substantially. The creation of a standardized mating and transfer interface would also allow multiple US and international launch companies to supply propellants to an orbital depot. These relatively dumb tankers would mostly consist of tanks sized to the launch vehicles that would be lofting them. A tanker could be built using similar construction to the launch vehicle's upper stage or even integrated directly into the upper stage ${ }^{32}$, with the addition of the mating and propellant transfer interface panel. The use of dumb tankers would permit a healthy amount of competition for propellant deliveries, which will provide a strong incentive for lowering prices over time.

\section{Near-term Propellant Depot Concepts}

While many depot concepts have been proposed which involve complex assembly operations on par with the contsruction of ISS, several innovative but simpler concepts have been recently proposed. These concepts provide enough capability to be immediately useful without requiring excessive up-front development, and can be evolved as the demand for higher flight-rates and additional services increase.

\section{A. ULA Disposable Single-Use "Pre-Depot" Concept}

The most straightforward propellant depot consists of a delivery tanker cryogenic supply tank, as in Fig. 6, modified with modest thermal insulation. Such a supply tank is designed specifically as part of a delivery tanker used for resupplying an orbital propellant depot. However, with modest thermal protection, such a tank can support months of near zero boil-off by taking advantage of the thermal capacitance of the launched propellant. This disposable "pre-depot" can be pre-launched and then used to directly supply propellant to a propulsion stage. To reduce development costs, the depot tank can be a derivative of existing upper stage tanks such as Centaur's $3 \mathrm{~m}$ diameter $\mathrm{LO}_{2}$ tank. Existing boosters, such as the Atlas V 551 or the RS-68A equipped Delta-IV Heavy, allow a "pre-depot" with as much as 20-26mT of $\mathrm{LO}_{2}$ capacity. For launch, the disposable "pre-depot" is stacked on the launch vehicle using standard payload interfaces.

To minimize heating, the tank has minimum penetrations. Avionics, docking port, reaction control system and fluid control are all mounted on the composite, low conductivity payload adapter. The entire system is shrouded in a thick MLI blanket, possibly further protected with a conic sunshield. By saturating the cryogenic propellant at atmospheric pressure prior to lift-off and allowing the bulk vapor pressure to slowly

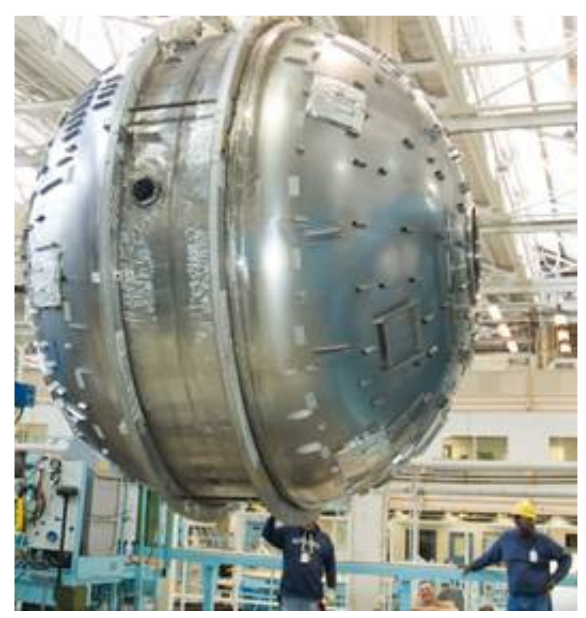

Figure 6. A Lightweight, Disposable "Pre-Depot" Tank, Derived from Centaur $\mathrm{LO}_{2}$ Tank, with $26 \mathrm{mT} \mathrm{\textrm {LO } _ { 2 }}$ Capacity. Credit ULA increase, the propellant can passively absorb tank heating for months with minimal need for tank venting. This period of minimal boil-off can be nearly doubled with pre-launch subcooling to just above the freezing point of the $\mathrm{LO}_{2}^{33}$. 
Use of this disposable "pre-depot" provides early, on orbit cryogenic propellant handling, storage and transfer experience allowing mission designers to gain confidence in the ability of depots to support complex exploration missions. This style of pre-depot enables highenergy missions with large payloads, such as Mars sample return, Europa landers, or lunar cargo ${ }^{34}$ or science landers using a dual-launch architecture, without the need for developing new HLVs. This architecture involves first launching $\mathrm{LO}_{2}$ in the disposable "pre-depot", followed by the propulsions stage, $\mathrm{LH}_{2}$ for the outbound trip, and the payload, as shown in Fig. 7.

\section{B. ULA Single-Fluid Single-Launch "Simple Depot" Concept}

A real depot, with longer-duration storage capabilities than the "pre-depot", and the ability to be refueled, has also been proposed by ULA. This simple depot, shown in Fig. 8 is composed of a 180" diameter cryogenic tank that can be launched inside of existing $5 \mathrm{~m}$ diameter payload fairings used by Atlas V, Delta IV or Ariane V. This depot would contain just a single fluid, either $140 \mathrm{mT}$ of LO2 or $15 \mathrm{mT}$ of LH2.

At the top of the depot is the hot equipment deck, which contains the avionics, control valves and station-keeping propulsion, and which connects to the docking collar. Sandwiched between the cold cryogenic propellant tank and the hot equipment deck is a low

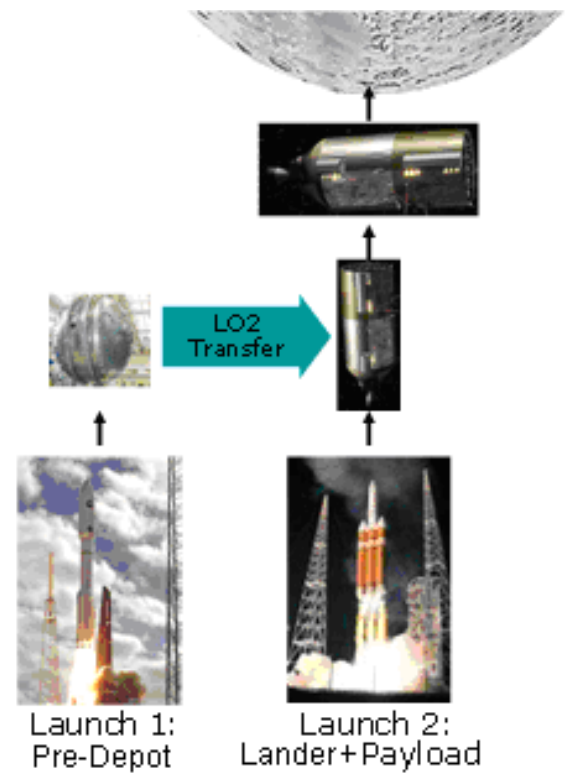

Figure 7. The Disposable "Pre-Depot" Concept Enables Heavy Lunar Landing Missions. Credit ULA

conductivity support truss and a thermal isolation gas reservoir. These structural elements provide a torturous thermal path to minimize heat transfer from the hot equipment deck to the propellant. Vapor cooling of the support truss can be used to further reduce tank heating. To minimize structural mass and maximize the depot propellant capacity within the payload fairing envelope, the isolation gas reservoir and the cryogenic propellant tank share a common, insulated bulkhead. The tank is enclosed in MLI to minimize Solar and Earth radiation heating. Once on orbit, a deployable sun shield cocoons the cold structure and cryogenic propellant tank, further reducing system heating, while allowing residual heat to radiate to deep space.

For launch, the deployable sun shield is stored on the equipment deck. Following separation from the launch vehicle the layers of sun shield are deployed. For a LEO depot, the deployed sun shield should form a concentric cone surrounding the depot. The depot maintains a northern ecliptic orientation, which enables the sun shield to shadow the tank from both solar and terrestrial heating throughout the LEO orbit. The multiple, concentric conical shield layers are maintained at different angles and provide an open path for thermal energy to be directed out into deep space, and away from the cryogenic propellant tank. Depots located in deep space, such as at EML-1 or EML-2, only encounter solar

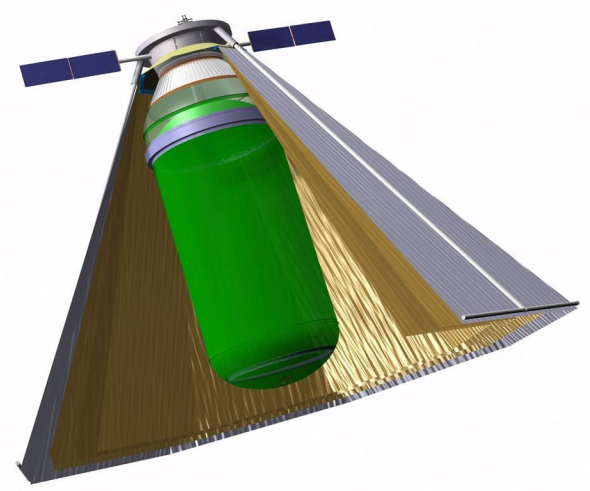

Figure 8. A Simple, Single-Fluid Depot Concept with Sunshield. Credit ULA heating and can use a planer sun shield similar to the James Webb Space Telescope.

The depot uses centrifugal propellant settling to simplify thermal control, liquid acquisition, and propellant transfer, without requiring the expenditure of reaction mass. Propellant transfer into and out of the depot is accomplished via differential pressure, similar to the way engines are fed on existing cryogenic stages. The wellinsulated depot can accommodate periods of zero-vent and no rotation to support operational needs such as docking.

The vented gas is stored in the large, cold gas thermal isolation reservoir. During quiescent operations, the reservoir is maintained at just below tank pressure allowing slow gas transfer from the propellant tank to maintain steady liquid propellant vapor pressure and, hence, temperature. This reservoir serves as the final heat sink between the hot equipment deck and the cryogenic propellant tank. The reservoir also supplies gas for the Reaction Control System (RCS) as well as positive pressure expulsion of liquids during propellant transfer.

There are many possible derivatives of this simple depot, which have a range of propellant storage capacities, but have progressively larger impacts on the launch vehicle and, therefore, on cost (see Figure 9). While a depot that fits entirely within the existing payload fairing, Fig. 9a, avoids the need to upgrade existing launch vehicles, it also severely restricts the depot's available propellant volume. A relatively low-cost launch vehicle upgrade, allowing 
greater depot propellant volume, could be accomplished by expanding the diameter of the depot to that of the payload fairing. This essentially integrates the depot tank and the payload fairing, as shown in Fig. 9b. Fig. 9c shows another option for increasing the depot volume, by integrating the depot directly with the upper stage. This option combines the available volume of the upper stage and payload fairing, providing the largest possible depot volume within the outer mould line of the existing launch vehicle. A much more intrusive option, shown in Fig. 9d, would be to develop a new, larger diameter upper stage for one of the existing launch vehicles such as Atlas $V$ or Delta IV. Past studies for both rockets have shown that their infrastructure can accommodate payload fairings diameters up to about $7 \mathrm{~m}$. Without developing a completely new rocket, this option would likely represent an upper limit on the volume of a single tank propellant depot.

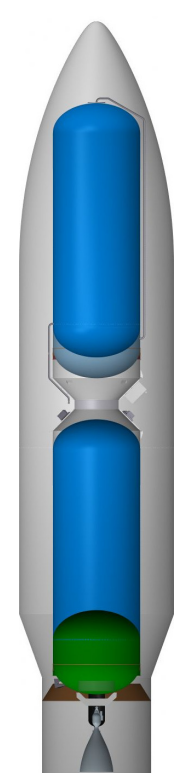

Depot as payload

$\mathrm{Vol}=60 \mathrm{~m}^{3}$

$M$ LO2 $=65 \mathrm{mT}$

$M \mathrm{LH} 2=4 \mathrm{mT}$

a)

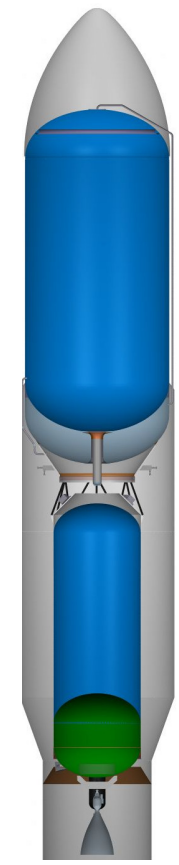

Depot as Payload Fairing $\mathrm{Vol}=200 \mathrm{~m}^{3}$ $M$ LO2 $=220 \mathrm{mT}$ $\mathrm{M} \mathrm{LH2}=14 \mathrm{mT}$

b)

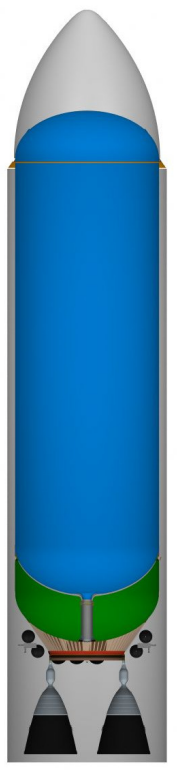

Combined depot/upper stage $\mathrm{Vol}=310 \mathrm{~m}^{3}$ $M$ LO2 $=350 \mathrm{mT}$ $\mathrm{M} \mathrm{LH} 2=22 \mathrm{mT}$

c)

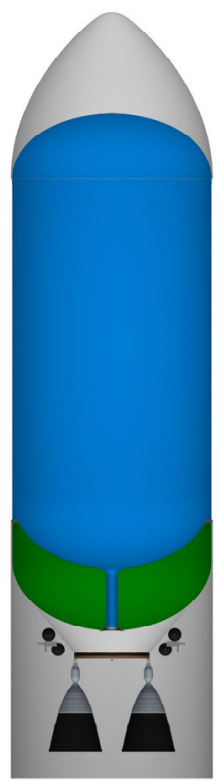

$7 \mathrm{~m}$ combined Depot/upper stag $€$ $\mathrm{Vol}=550 \mathrm{~m}^{3}$ $M L O 2=610 \mathrm{mT}$ $M \mathrm{LH} 2=38 \mathrm{mT}$

d)

Figure 9. Several Options for Single-Launch, Single-Fluid Depots Using Existing Launch Vehicle Payload Fairings. Credit ULA

\section{Dual-Fluid Single-Launch Depot Concept}

While a single-fluid depot can enable many missions that would otherwise have required a Super Heavy Lift Vehicle (SHLV), the ability to replenish both propellants at an orbital depot truly opens the Solar System to robust exploration. A concept for a simple, near-term, single-launch $\mathrm{LO}_{2} / \mathrm{LH}_{2}$ depot with sufficient capacity to support manned spaceflight beyond LEO has been independently conceived by several of this paper's authors. This depot would consist of an integrated assembly with a large $\mathrm{LH}_{2}$ depot tank connected to a modified upper stage, which would be converted to an $\mathrm{LO}_{2}$ tank after delivering the assembly to orbit. The depot would use rotation to provide centrifugal settling during quiescent operations, except during docking of visiting vehicles, where the station might de-spin to simplify docking operations.

The $\mathrm{LH}_{2}$ tank would be constructed in a similar manner to the single-fluid concept shown above in Figure $9 \mathrm{~b}$. The tank would be integral with the $5 \mathrm{~m}$ payload fairing wall, in order to maximize its propellant capacity without requiring changes to the launch vehicle outer mold-line. The tank would include a small vapor reservoir between the main tank and the depot equipment deck which would interconnect the two halves of the depot. The equipment deck would include the attachment point for the sunshield, would hold solar panels, and house the valves and 
controls for the $\mathrm{LH}_{2}$ half of the depot. The depot equipment deck would be attached to the $\mathrm{LH}_{2}$ tank and the $\mathrm{LO}_{2}$ tanks via low-conductivity materials.

The $\mathrm{LO}_{2}$ half of the depot would be constructed by adding several mission-specific modifications to the upper stage used to orbit the vehicle. These additions would include MLI to provide in-space thermal insulation, docking adapters and transfer interfaces mounted on the aft end of the stage, and some additional plumbing and controls for depot operations. The stage would be converted to $\mathrm{LO}_{2}$ use after arriving at the destination orbit by first transferring any remaining $\mathrm{LH}_{2}$ from the upper stage $\mathrm{LH}_{2}$ tank into the much larger depot $\mathrm{LH}_{2}$ tank. The upper stage $\mathrm{LH}_{2} \operatorname{tank}$ would then be vented to verify that no residual hydrogen remained. After allowing it to sit open to vacuum for some time, the tank would be resealed and any remaining $\mathrm{LO}_{2}$ from the upper stage would be transferred from the upper stage $\mathrm{LO}_{2}$ tank into the now-empty upper stage $\mathrm{LH}_{2}$ tank. The emptied upper stage $\mathrm{LO}_{2}$ tank would then serve as the gas barrier to insulate the $\mathrm{LO}_{2}$ tank from heat flowing out of the aft section. For thermal control, the $\mathrm{LO}_{2}$ section would take advantage of the fact that $\mathrm{LH}_{2}$ has a heat capacity ten times higher than $\mathrm{LO}_{2}$. By using the boiled hydrogen to chill the $\mathrm{LO}_{2}$ tank and the interconnects between the tanks and hot structures, the depot would be able to completely suppress $\mathrm{LO}_{2}$ boil-off, even though the $\mathrm{LO}_{2}$ section would not include its own sunshield, and in spite of the rather severe thermal environment in LEO. As mentioned previously, the $\mathrm{LH}_{2}$ boil-off in this situation is still less than the reaction mass requirements for station-keeping, so none of the boil-off $\mathrm{LH}_{2}$ is actually wasted.

An illustration of such a depot, based on the ULA ACES upper stage ${ }^{35}$ is shown below in Fig. 10. Using the ACES stage, the depot would hold $121 \mathrm{mT}$ of propellant (106 $\mathrm{mT}$ of $\mathrm{LO}_{2}$ and $15 \mathrm{mT}$ of $\mathrm{LH}_{2}$ ). It should be noted however, that this concept could also be based on existing stages such as the Centaur or Delta-IV Heavy upper stages, or other proposed $\mathrm{LO}_{2} / \mathrm{LH}_{2}$ upper stages such as SpaceX's Raptor, or Arianespace's Ariane 5 ESC-B. A depot using a stock Centaur as its $\mathrm{LO}_{2}$ tank would be able to hold about $52 \mathrm{mT}$ of $\mathrm{LO}_{2}$ and $14 \mathrm{mT}$ of $\mathrm{LH}_{2}$. The resulting oxidizer to fuel $(\mathrm{O} / \mathrm{F})$ ratio is 3.7 , which is far richer than the 5.5-6:1 ratio typical for existing upper stages, in which only about $62 \mathrm{mT}$ of propellant would be usable. However, by stretching the Centaur stage $\mathrm{LH}_{2} \operatorname{tank}$ by about $1.5 \mathrm{~m}$ (and shortening the depot tank by the same amount to keep it within the boundaries of the existing fairings), the total propellant loads become about $64 \mathrm{mT}$ of $\mathrm{LO}_{2}$ and $12 \mathrm{mT}$ of $\mathrm{LH}_{2}$, giving a more useful $\mathrm{O} / \mathrm{F}$ ratio of 5.4. This would leave some extra $\mathrm{LH}_{2}$ tankage to handle the higher boil-off. Tank barrel stretches are far less expensive than changes to the diameter of the tanks, which require redesigning the complicated aft-end of the rocket, new tooling and qualification testing. In fact, many of the upgrades to the Centaur stage over the years have consisted of such barrel stretching ${ }^{36}$.

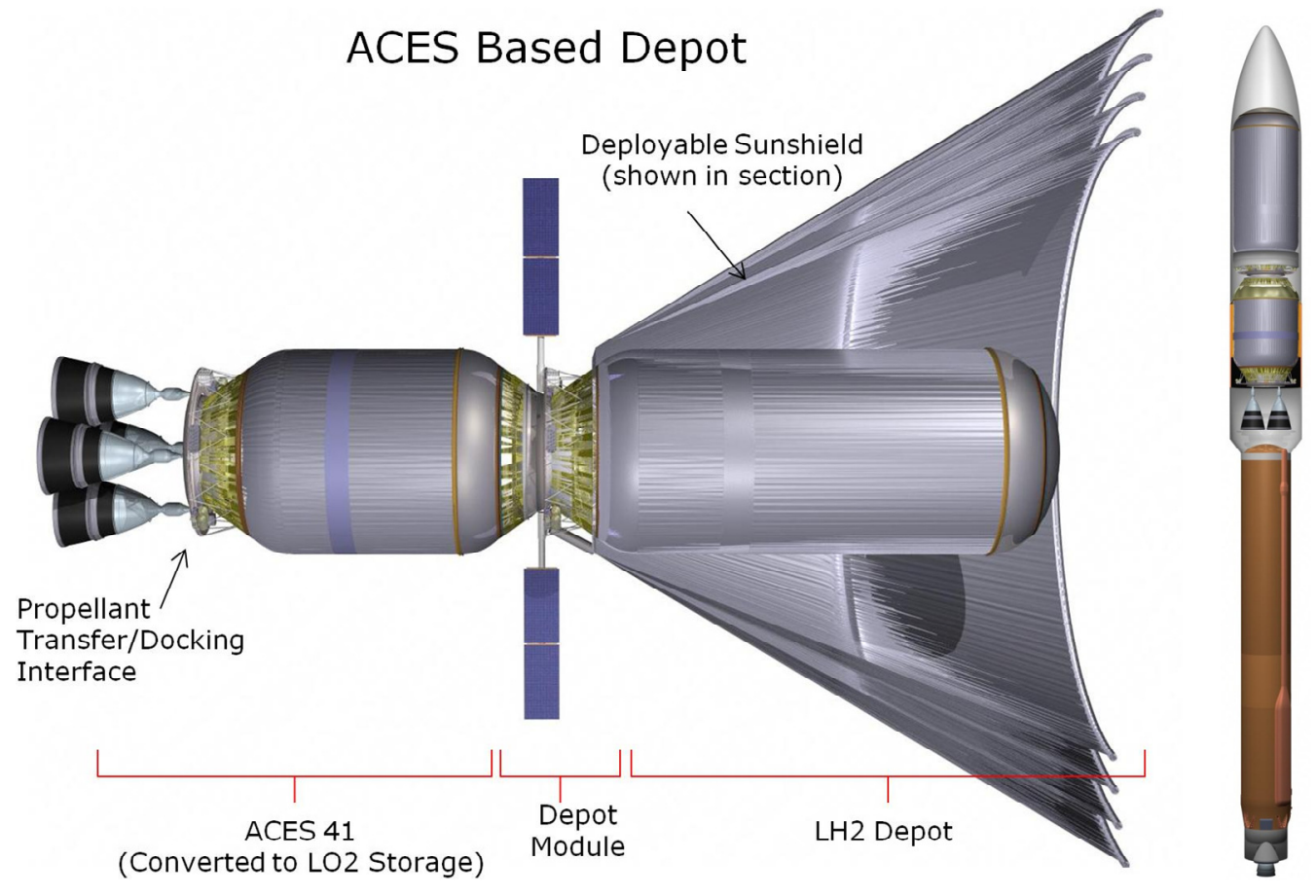

Figure 10. A Single Launch, Dual-Fluid Propellant Depot. Credit ULA 
By combining a depot tank with a propulsive stage, this depot concept is able to self-deploy to locations beyond LEO, such as at EML-1 or -2, or even as far as Mars orbit. By placing one of these depots in LEO and one in either EML-1 or -2, ESAS-class lunar missions can be performed without requiring vehicles bigger than existing launchers, and without requiring a new Earth departure stage. The severe thermal environment in LEO causes a substantial amount of propellant boil-off over the course of a year. EML-1 or -2 depots are in a much more benign thermal environment, with very low boil-off levels. This leads to the conclusion that the best way to use a depot system like this is to forward propellants on from the LEO depot to the EML-1 or -2 depot as quickly as possible. The higher the tempo of flights beyond LEO, the lower the percentage of propellants lost to boil-off in LEO. With a decent operational tempo, boil-off losses for this system can be kept to low single-digit percentages of the yearly propellant throughput.

\section{Boeing Multi-Launch Dual-Fluid Depot Concept}

The Boeing propellant depot architecture, shown below in Fig. 11, would include two independent depots in LEO, a reusable propellant carrier and a low-cost launch vehicle, such as the SpaceX Falcon 9. Each depot would consist of a central truss and six tank modules derived from the Delta IV Heavy upper stage. Each depot would be sized to provide sufficient propellant to fill the ESAS Reference Architecture LSAM DM and to replenish the EDS propellant used during ascent ${ }^{37}$.

The truss and empty tank modules would be launched individually on Falcon 9 launch vehicles. Each tank module has a capacity of $25 \mathrm{mT}$. Propellant would be delivered to the depot by reusable propellant carriers with a capacity of $9.4 \mathrm{mT}$ each. Propellant carriers would be berthed to the propellant transfer port on the depot truss. A robotic arm removes and releases the propellant carrier following propellant transfer. Propellant carriers would be able to be used a maximum of 10 times before being replaced. A reusable transfer stage is included in the growth plans for the Boeing Depot Architecture.

A multiple-tank configuration depot with central truss was selected based on Boeing's trade study of 13 different concepts. ${ }^{38}$ Atlas V and Delta IV upper stages were considered as depot tank modules. The Delta IV Heavy upper stage configuration was chosen because the depot capacity requirement could be met with six tank sets instead of the eight required if the stock Atlas V Centaur upper stage were used. Propulsion and avionics system modifications along with additional thermal protection and micrometeoroid and orbital debris shielding were defined and mass properties estimated.

The depot modules would incorporate Orbital Express autonomous capabilities for rendezvous and proximity operations.. The truss would include two robotic arms to berth depot storage tanks, propellant carriers and EDS to appropriate locations.

Propellant depot capacity was defined by the LSAM DM propellant capacity and the EDS propellant used during ascent. LSAM DM propellant mass, as studied by the NASA ESAS team, varied between 25 and $30 \mathrm{mT}^{39}$. Boeing estimated LSAM DM propellant mass to be $25 \mathrm{mT}$ based on the ESAS CaLV Case 2 mass allocation ${ }^{40}$. The EDS contained 490,744 lbm (222.6mT) at lift-off and 219,443 lbm $(99.5 \mathrm{mT})$ remained upon reaching LEO. Therefore, a LEO propellant depot would have to provide a minimum of $147 \mathrm{mT}$ to the EDS and LSAM DM.

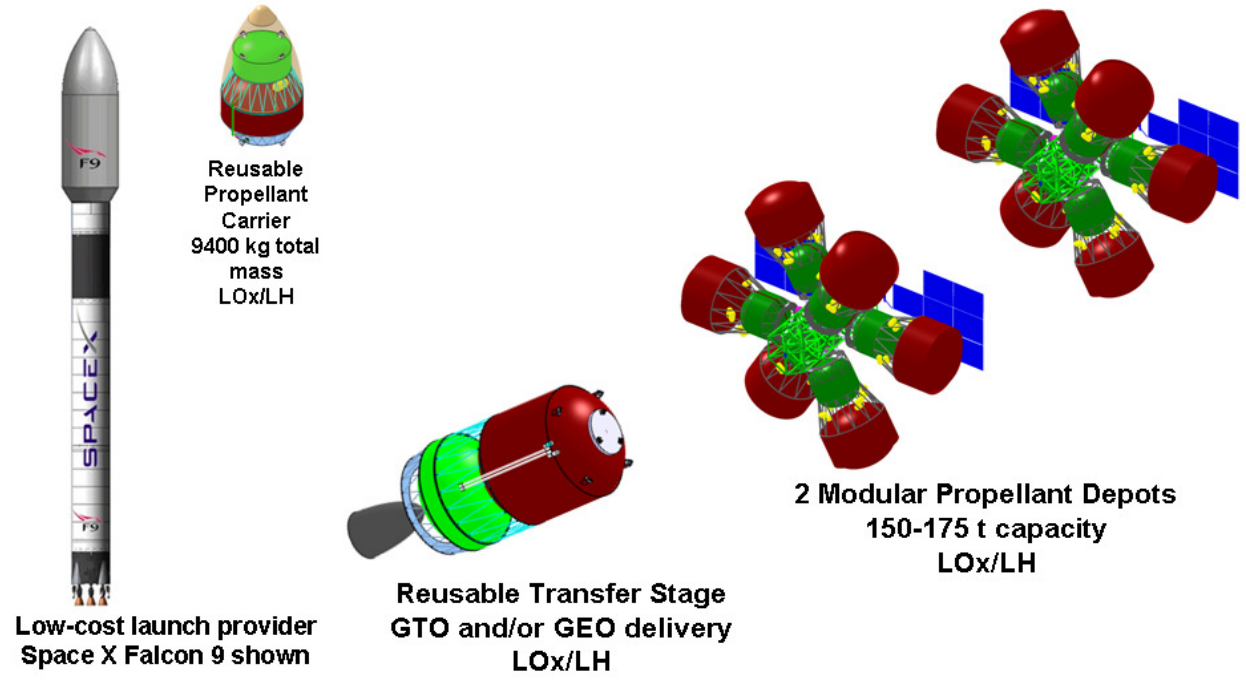

Figure 11. Elements of the Boeing Modular Depot Concept. Credit Boeing 


\section{E. Comparison of Near-Term Depot Concepts}

Below in Table 2, the relative strengths of the four depot concepts described in this section are compared.

Table 2. A Comparison of Four Near-Term Depot Concepts

\begin{tabular}{|c|c|c|}
\hline Depot Concept & Advantages & Disadvantages \\
\hline Disposable Pre-Depot & $\begin{array}{l}\text { - Very simple system, high TRL } \\
\text { - Shares some commonality with } \\
\text { tanker design for reusable depots } \\
\text { - Single launch, no orbital assembly } \\
\text { - No on-going operation expenses for } \\
\text { infrequent mission use }\end{array}$ & $\begin{array}{l}\text { - Only provides one fluid, typically } \mathrm{LO}_{2} \text {, } \\
\text { requires } \mathrm{LH}_{2} \text { brought with transfer stage } \\
\text { - } \quad \text { Limited depot size }<26 \mathrm{mT} \mathrm{LO}_{2} \\
\text { - } \quad \text { Limited to medium-term storage } \\
\text { - } \text { Barely capable of fueling minimalist } \\
\text { manned missions beyond LEO using } \\
\text { existing launchers } \\
\text { - } \quad \text { eepot in mission critical path }\end{array}$ \\
\hline $\begin{array}{l}\text { Single-Fluid Simple } \\
\text { Depot }\end{array}$ & $\begin{array}{l}\text { - Large depot capacity, } 200 \mathrm{mT} \mathrm{LO}_{2} \\
\text { - Single launch, no orbital assembly } \\
\text { - } \mathrm{LO}_{2} \text {-only operations are thermally } \\
\text { much easier in LEO than } \mathrm{LH}_{2} \text { storage } \\
\text { - Capable of zero boil-off operations } \\
\text { at EML-1 or -2. }\end{array}$ & $\begin{array}{l}\text { - Only provides one fluid, typically } \mathrm{LO}_{2} \text {, } \\
\text { requires } \mathrm{LH}_{2} \text { brought with transfer stage or } \\
\text { second simple depot } \\
\text { - Restricts beyond-LEO manned } \\
\text { missions with performance of existing } \\
\text { stages } \\
\text { - Depot in mission critical path }\end{array}$ \\
\hline $\begin{array}{l}\text { Dual-Fluid Single- } \\
\text { Launch Depot }\end{array}$ & $\begin{array}{l}\text { - Medium depot capacity, } 76-117 \mathrm{mT} \\
\text { of LO2/LH2 } \\
\text { - Single launch, no orbital assembly } \\
\text { - Self-deployable to almost anywhere } \\
\text { in the inner Solar System } \\
\text { - Allows re-tanking of both upper } \\
\text { stage propellants } \\
\text { - Enables ESAS-class and larger } \\
\text { manned lunar missions using existing } \\
\text { launch vehicles, with depots in LEO } \\
\text { and at EML-1 or -2 } \\
\text { - All } \mathrm{LO}_{2} / \mathrm{LH}_{2} \text { can be delivered by } \\
\text { small launch vehicles or RLVs over as } \\
\text { many flights as makes economic sense } \\
\text { - Depot and propellant launch not in } \\
\text { mission critical path }\end{array}$ & $\begin{array}{l}\text { - } \mathrm{LH}_{2} \text { use in } \mathrm{LEO} \text { causes high boiloff } \\
\text { depore complicated than the single-fluid } \\
\text { - Requires significant modifications to } \\
\text { the depot launcher upper stage. } \\
\text { - Extra operational complications and } \\
\text { risk due to reusing upper stage } \mathrm{LH}_{2} \text { tank } \\
\text { for } \mathrm{LO}_{2} \text { storage. } \\
\text { - Large station has substantial station- } \\
\text { keeping requirements }\end{array}$ \\
\hline $\begin{array}{l}\text { Multi-Launch Modular } \\
\text { Depot }\end{array}$ & $\begin{array}{l}\text { - Large depot capacity, } 150 \mathrm{mT} \\
\mathrm{LO}_{2} / \mathrm{LH}_{2} \text { and larger } \\
\text { - Integral robotic arm makes berthing } \\
\text { of visiting vehicles much easier } \\
\text { - Capable of zero boil-off operations } \\
\text { or at least very low boil-off. } \\
\text { - Could be combined with the dual- } \\
\text { fluid design above to yield very large } \\
\text { propellant depots, }>450 \mathrm{mT} \mathrm{LO}_{2} / \mathrm{LH}_{2} \\
\text { capability } \\
\text { - Depot and propellant launch not in } \\
\text { mission critical path }\end{array}$ & $\begin{array}{l}\text { - Requires multiple launches } \\
\text { - Requires orbital assembly (albeit } \\
\text { mostly autonomous) } \\
\text { - Large station has substantial station- } \\
\text { keeping requirements }\end{array}$ \\
\hline
\end{tabular}

\section{Orbital and Suborbital Tools for Depot Technology Demonstration}

While the introduction of new technologies can lead to dramatic improvements in the affordability of space programs, incorporating new technologies always carries technical risks, and aggressive early retirement of those risks is a key to avoiding programmatic cost-overruns and delays. One of the most important steps in this process of 
space technology maturation, especially for systems involving complicated phenomena like cryogenic fluid management, is flight testing in the space environment. ${ }^{41}$ Unfortunately, this step is often hampered by the high cost and infrequent opportunities for flight testing. In many cases this prevents adequate experimentation with alternative approaches to truly evaluate their feasibility. Space architectures often suffer thereby from conceptual lock-in, where judgment decisions made during early phases with marginal and incomplete data win out over promising new concepts ${ }^{42}$. Recent progress in developing orbital testbeds for cryogenic fluid management and in the fielding of commercial reusable suborbital vehicles means that a wider range of technological solutions can now be affordably and extensively tested. These capabilities allow various propellant depot technologies to be rapidly matured, while simultaneously increasing the probability that promising alternative technological approaches will be adequately investigated as well.

\section{A. CRYOTE}

In order to provide a method for flight-testing promising cryogenic technologies in space, ULA has partnered with NASA and industry to develop the Cryogenic Orbital Testbed (CRYOTE) ${ }^{43}$. This system, shown in Fig. 12 and 13, consists of a large experimental cryogenic tank integrated into the EELV Secondary Payload Adapter (ESPA) ring, with the valves and controls for the testbed located at one of the six secondary payload locations. CRYOTE is designed to fly as a secondary payload on Atlas V missions, thus increasing affordability and frequency of flight opportunities. The $\mathrm{LH}_{2}$ used for the testbed is transferred from the Centaur $\mathrm{LH}_{2}$ tank after delivery of the primary payload to its destination orbit, thus avoiding any risk to the success of the primary mission.

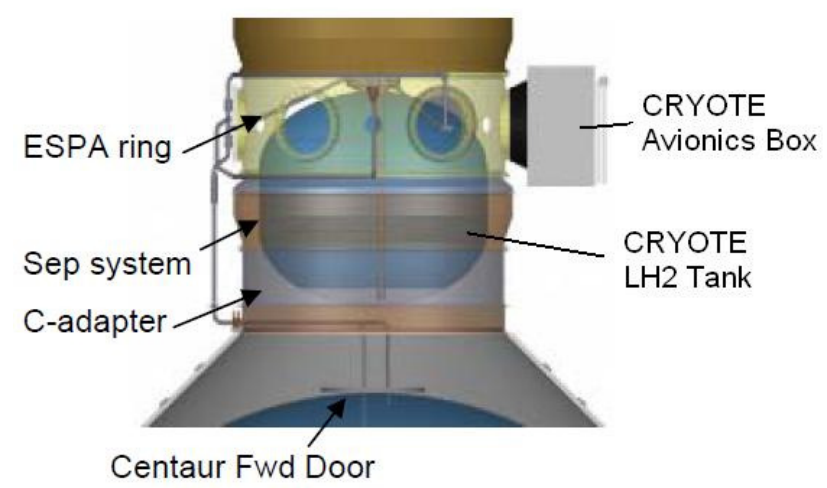

Figure 12. CRYOTE System Elements. Credit ULA

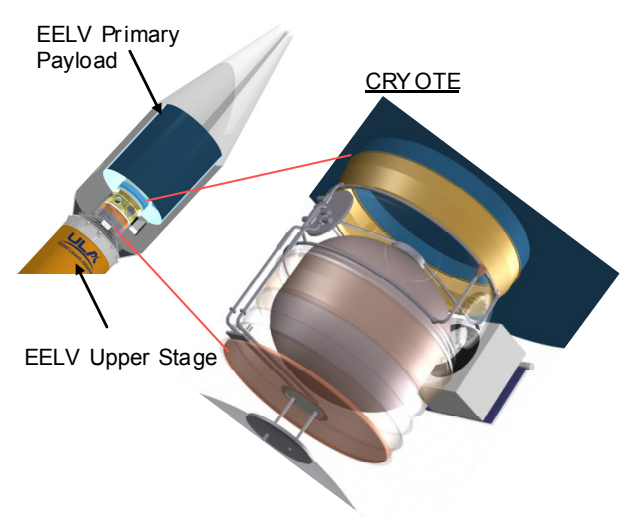

Figure 13. CRYOTE on Atlas V. Credit ULA

CRYOTE provides a platform for testing a wide range of technologies needed for depots, cryogenic landers, and long-duration cryogenic in-space stages. These technologies include active and passive thermal control technologies, various propellant settling techniques, liquid acquisition and mass gauging techniques, and propellant transfer. The very large size of the CRYOTE tank, compared with earlier proposed ${ }^{44}$ and historical cryogenic fluid management testbeds, allows for more realistic testing of cryogenic technologies on a scale where surface tension and surface area to volume ratios are closer to those in a real depot.

The CRYOTE system is currently funded, and ground test articles are being designed and fabricated by Innovative Engineering Solutions of Murrietta, CA with assistance from ULA and NASA. Depending on funding, CRYOTE may be ready for its first flight as soon as 2012.

\section{B. Suborbital RLVs As Space Technology Testbeds}

CRYOTE provides a very important method for flight-demonstrating key technologies for propellant depots. However, the frequency of flight opportunities for CRYOTE may not be able to fly the large number of existing cryogenic technology concepts, meaning that it only partially resolves the challenge of providing an environment where depot technology can be adequately explored. Also, flight demonstrations and experiments often become unplanned learning experiences, requiring additional follow-up experiments to resolve issues that can only be discovered by actual flight testing. Suborbital RLVs can help solve this challenge and provide a useful complement to orbital testbeds like CRYOTE.

Suborbital RLVs, such as those under development by Masten Space Systems, Blue Origin, Armadillo Aerospace, Scaled Composites, and XCOR Aerospace, are capable of frequent flights of experimental payloads to 
the edge of space, providing a few minutes of microgravity per flight. These vehicles are designed to return to their launch site, where experiments can be recovered and even reflown same-day, if necessary. Masten Space Systems, in particular, is investigating the feasibility of developing a suborbital version of the CRYOTE system for flight on its XA-1.0 vehicle (shown in Fig. 14). Vertical takeoff and landing systems, such as those being developed by MSS, Blue Origin, and Armadillo, are particularly useful for cryogenic fluid management experiments.. Most are capable of being flown unmanned, and they tend to have spacious payload accommodations because wide diameters are needed for landing stability. .Unmanned flight capability may be important for flights involving highly flammable liquids like $\mathrm{LH}_{2}$, because it allows the cabin atmosphere to be filled with a neutral buffer

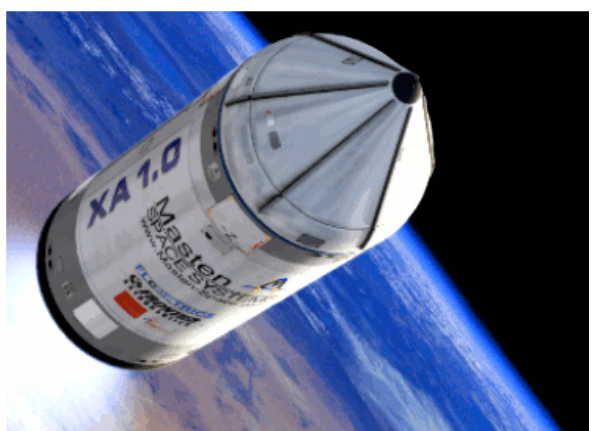

Figure 14. XA-1.0 Suborbital RLV. Credit MSS

gas like helium. The high flight rate capability of these vehicles, combined with the lower cost of accessing suborbital space makes it easier to explore a wider range of alternative depot-enabling technologies. Also, by substantially lowering the cost of failure, suborbital RLVs allow experimentation to be done in a rapid, iterative process, as is typical in non-space technology development projects. By trying out technologies and experimental hardware earlier in the development process, knowledge can be gained less expensively and costly detours can be avoided.

Suborbital vehicles do not provide the same long-duration flight capability that may be necessary to ultimately demonstrate a complete, integrated depot system. However, by allowing earlier experimentation, and by allowing CRYOTE payloads to be tested out before being committed to an expensive orbital mission, they can provide a great enhancement to the capabilities provided by CRYOTE.

\section{Conclusion}

While there is still work to be done to bring orbital propellant depots into reality, the technology is at the point where it can be incorporated into manned space transportation systems and be moved forward. The depot design concepts discussed in this paper offer realistic, near-term options that would be useful in a wide variety of manned exploration missions, and would enable commercial manned spaceflight beyond LEO. The tools being developed for flight-testing and maturing these propellant depot technologies make propellant depots much closer to reality than they have ever been. Depots are a key capability for a spacefaring civilization that are ready for development today.

\section{References}

${ }^{1}$ Amundsen, R. "The South Pole: An Account of the Norwegian Antarctic Expedition in the 'Fram', 1910-1912" Interlink Publishing Group, 2003. Chapter VI: Depot Journeys.

2 Stone, W. C., "The Challenge of the Pena Colorada," No. 14, September 1984, pp. 46-55, AMCS Activities Newsletter, Association for Mexican Cave Studies, Box 7672, Austin, TX 78713

${ }^{3}$ Sykora, Fritz, "Guido von Pirquet-Austrian Pioneer of Astronautics," History of Rocketry and Astronautics, R. Cargill Hall, ed., AAS Publications, San Diego, 1986, p. 151.

${ }^{4}$ Wilson, D. J.; Hale, D. V.; Whitacre, W. E. "Orbital propellant depot system" (Cryogenic Engineering Conference, Boulder, Colo., June 17-19, 1970.) In: Advances in cryogenic engineering. Volume 17 (A72-3882619-33) New York, Plenum Press, 1972, p. 160-165.

${ }^{5}$ Flemming, Ken. "STV fueling options" in NASA, Washington, Beyond the Baseline 1991: Proceedings of the Space Station Evolution Symposium. Volume 1: Space Station Freedom, Part 2 p 489-531 (SEE N92-17409 08-18)

${ }^{6}$ Von Braun, W. "Concluding Remarks by Dr. Wernher von Braun about Mode Selection for the Lunar Landing Program", 7 June 1962, Lunar-Orbit Rendezvous File, NASA Historical Reference Collection, NASA Headquarters, Washington D.C. p. 7

${ }^{7}$ Kutter, Bernard. "Commercial Launch Services: An Enabler for Launch Vehicle Evolution and Cost Reduction", AIAA-2006-7271.

${ }^{8}$ Goff, J., Traugott, S., and Oesterle, A. "Depot-Centric Human Spaceflight". Whitepaper submitted to the Review of US Human Spaceflight Plans Committee, July 2009.

http://www.nasa.gov/pdf/376490main_026\%20-\%2020090722.2.Depot-Centric_Human_Spaceflight.pdf

${ }_{9}^{9}$ Blair, J.C., Ryan, R.S., Schutzenhofer, L.A., Humphries, W.R. "Launch Vehicle Design Process: Characterization,

Technical Integration, and Lessons Learned". NASA/TP-2001-210992. p. 35. 
${ }^{10}$ Hansen, James R., "Enchanted Rendezvous, "Monographs in Aerospace History, Series \#4, NASA Headquarters History Office, January 1999, pp. 30.

${ }^{11}$ LeBar, J., and Cady, E., "The Advanced Cryogenic Evolved Stage (ACES)—A Low-Cost, Low-Risk Approach to Space Exploration Launch”. AIAA-2007-7454. September, 2007.

http://www.ulalaunch.com/docs/publications/TheAdvancedCryogenicEvolvedStageACES2006LeBar7454.pdf

12 Jeffrey S. De Kruif, “Centaur Upperstage Applicability for Several-Day Mission Durations with Minor Insulation Modifications”, AIAA-2007-5845, July 2007,

http://www.ulalaunch.com/docs/publications/CentaurUpperstageApplicabilityforSeveralDayMissionDurationswith MinorInsulationModificationsAIAA20075845.pdf.

${ }^{13}$ Bernard Kutter, "A Practical, Affordable Cryogenic Propellant Depot based on ULA's Flight Experience”, AIAA 2008-7644, September 2008, http:/www.ulalaunch.com/docs/publications/APracticalAffordableCryogenicPropellantDepotBasedonULAsFlightE xperience20087644.pdf

${ }^{14}$ Bonometti, J., Sorensen, K., Jansen, R., Dankanich, J., and Frame, K. "Free Re-boost Electrodynamic Tether on the International Space Station". AIAA-2005-4545. July 2005.

${ }^{15}$ Marchetta, J.G., Simmons, B.D., Hochstein, J.I., "Magnetic Retention of $\mathrm{LO}_{2}$ in an Accelerating Environment," Acta Astronautica, Vol. 62, 8-9, pp.478-490, April-May 2008.

${ }^{16}$ Martin, J.J., Holt, J.B. "Magnetically Actuated Propellant Orientation Experiment, Controlling Fluid Motion with Magnetic Fields in a Low-Gravity Environment." NASA TM 210129.

${ }^{17}$ Marchetta, J.G., Roos, K.M., "Simulating Magnetic Positive Positioning of Cryogenic Propellants in a Transient Acceleration Field,"Computers and Fluids, Sept. 2008,DOI: 10.1016/j.compfluid.2008.09.005.

${ }^{18}$ Marchetta, J.G., "Simulation of LOX Reorientation Using Magnetic Positive Positioning," Microgravity Science and Technology Journal, Vol. 18, pp. 31-39, 2006.

${ }^{19}$ Marchetta J.G., Hochstein, J.I., "Simulation and Prediction of Magnetic Propellant Reorientation in Reduced Gravity", AIAA Journal of Propulsion and Power, Vol. 20, no. 5, pp.927-935, September-October 2004.

20 J. J. Martin, "Large-Scale Liquid Hydrogen Testing of a Variable Density Multilayer Insulation With a Foam Substrate", NASA/TM-2001-211089, June 2001.

${ }^{21}$ Kutter et al. "Atlas Centaur Extensibility to Long-Duration In-Space Applications”, AIAA 2005-6738, Sept 2005, http://www.ulalaunch.com/docs/publications/AtlasCentaurExtensibilitytoLongDurationInSpaceApplications200567 38.pdf

${ }^{22}$ Dew, M. et al., "Design and Development of an In-Space Deployable Sun Shield for the Atlas Centaur", AIAA2008-7764, September 2008,

http://www.ulalaunch.com/docs/publications/DesignandDevelopmentofanInSpaceDeployableSunShieldfortheAtlasC entaur20087764.pdf

${ }^{23}$ Kirk Allwein, “Atlas Centaur Sun Shield Design and Testing, An Update”, AIAA 2009-6587, September 2009.

24 Ewing, A., Back, J., Schuettpelz, B., and Laue, G. "James Webb Space Telescope Sunshield Membrane Assembly". AIAA-2009-2156. May 2009.

${ }^{25}$ Chato, D. "Technologies for Refueling Spacecraft on Orbit”. NASA/TM-2000-210476. November 2000.

${ }^{26}$ Friend, R. B., "Orbital Express Program Summary And Mission Overview”, 2008 6958-02

${ }^{27}$ Mulder, T.A., “Orbital Express Autonomous Rendezvous and Capture Flight Operations”, Air Force T\&E Days 2008, Los Angeles CA.

${ }^{28}$ Dipprey, N. F., et al., Orbital Express Propellant Resupply Servicing, $39^{\text {th }}$ AIAA/ASME/SAE/ASEE JPC, AIAA 2003-4898

${ }^{29}$ Dean, M., Zegler, F. “Slip Joint Duct System”. US Patent \#6,449,942 B1, granted September 2002.

${ }^{30}$ Michael Johnson, "Astrotech Research \& Conventional Technology Utilization Spacecraft (ARCTUS)", AIAA2007-6130, September 2006.

${ }^{31}$ Charles Miller, “A Public Offer of Near-Term, Low-Risk, Low-Cost ISS Cargo Delivery Service on US Launch Vehicles”. Newspace 2006, July 2006. http://www.constellationservices.com/csi_publicoffer_isscargo_jul06.pdf

${ }^{32}$ Frank Zegler, "A Commercially Based Lunar Architecture”,AIAA-2009-6567, September 2009

${ }^{33}$ Shuvo Mustafi, "Subcooling Cryogenic Propellants for Long Duration Space Exploration", AIAA-2009-6584, September 2009

34 Bernard F. Kutter, "Robust Lunar Exploration Using an Efficient Lunar Lander Derived from Existing Upper Stages”, AIAA-2009-6566, September 2009

${ }^{35}$ Mark Wilkins, “Upper Stage Evolution”, JPC 2009, August 2009, http://www.ulalaunch.com/docs/publications/UpperStageEvolutionJPC2009.pdf 
${ }^{36}$ Thomas J. Rudman, "The Centaur Upper Stage Vehicle", $4^{\text {th }}$ IAC on Launcher Technology, December 2002, http://www.ulalaunch.com/docs/publications/TheCentaurUpperStageVehicleHistory.pdf

${ }^{37}$ Bienhoff, D, "The Potential Impact of a LEO Propellant Depot On the NASA ESAS Architecture," Space Technology \& Applications International Forum (STAIR-2007), Albuquerque, NM, 11-15 February, 2007

${ }^{38}$ Chandler, F., Bienhoff, D., Cronick, J., and Grayson, G. "Propellant Depots for Earth Orbit and Lunar Exploration". AIAA-2007-6081. September 2007.

${ }^{39}$ NASA Exploration System Architecture Final Report, Tables 4-21 and 4-23

http://www.nasa.gov/pdf/140635main_ESAS_04.pdf

${ }^{40}$ NASA Exploration System Architecture ESAS Final Report Figure 6-43

http://www.nasa.gov/pdf/140637main_ESAS_06.pdf

${ }^{41}$ Chato, D., "The Role of Flight Experiments in the Development of Cryogenic Fluid Management Technologies". NASA/TM-2006-214261. http://gltrs.grc.nasa.gov/reports/2006/TM-2006-214261.pdf

${ }^{42}$ Bonometti, J. "Boom Rendezvous Alternative Docking Approach". AIAA-2006-7239. September 2006.

${ }^{43}$ Mari Gravlee, "CRYOTE (Cryogenic Orbital Test) Concept", AIAA-2009-6440, September 2009

${ }^{44}$ Sakla, S., Kutter, B., and Wall, J. "Centaur Test Bed (CTB) for Cryogenic Fluid Management". AIAA-2006-4603. http://www.ulalaunch.com/docs/publications/CentaurTestBedCTBforCryogenicFluidManagement20064603.pdf 